\title{
FREE COMPLETELY DISTRIBUTIVE LATTICES
}

\author{
GEORGE MARKOWSKY ${ }^{1}$
}

\begin{abstract}
We show that the usual construction of the free distributive lattice on $\boldsymbol{n}$ generators generalizes to an arbitrary quantity of generators and actually yields a free completely distributive lattice. Furthermore, for an infinite number of generators the cardinality of the corresponding free completely distributive lattice is exactly that of the power set of the power set of the set of generators.
\end{abstract}

Free distributive lattices over a finite number of generators have interested a number of authors over a long period of time (see [1], [4], [5], and [6] for references). For a finite set, $G$, of $n$ generators it is well-known that the free distributive lattice over $n$ generators is isomorphic to the collection of all closed below subsets of the power set of $G$. It is also possible to find free distributive lattices over an infinite number of generators [7]. However, these are quite different objects.

For a given set $X$ of arbitrary cardinality, we denote by $F D(|X|)$ the collection of all closed below subsets of the power set $\left(2^{X}\right)$ of $X$. We then have the following result.

TheOrem 1. Let $X$ be $a$ set and $I: X \rightarrow F D(|X|)$ be given by $I(x)=\{T \subset$ $X \mid x \notin T\}$. Then I is well-defined and injective and for any completely distributive lattice $L$ and map $\Theta: X \rightarrow L$, there exists a unique lattice homomorphism $\Theta^{*}: F D(|X|) \rightarrow L$, such that $\Theta^{*}$ preserves all sups and infs and $\Theta^{*} I=\Theta$. (Note that when we say that a lattice is completely distributive, we mean to imply that it is complete.)

Proof (sketch): Let $\Theta^{*}$ be given by $\Theta^{*}(T)=\sup _{S \in T^{\prime}}\left\{\inf _{X \in S} \Theta(x)\right\}$ where $T^{\prime}=\{X-S \mid S \in T\}$. Now verify that $\Theta^{*}$ has all the properties asserted above.

It follows in the usual manner that $F D(|X|)$ is unique up to isomorphism. This fact and Theorem 1 justify calling $F D(|X|)$ the free completely distributive lattice on $|X|$ generators. It is interesting to note that Gaifman [2] and Hales [3] have shown that there does not exist a Boolean algebra over countably many generators. For finite sets $X$, the free distributive lattice on $|X|$ generators is often considered to be our $F D(|X|)-\left\{\varnothing, 2^{X}\right\}$ (see [1]). However, to make the mapping property hold we need $\varnothing$ and $2^{X}$, since any

Received by the editors February 23, 1978.

AMS (MOS) subject classifications (1970). Primary 06A25.

Key words and phrases. Free distributive lattice, free completely distributive lattice.

${ }^{1}$ Supported in part by ONR Contract N00014-67-A-0298-0015. 
sup and inf preserving mapping between complete lattices maps least elements to least elements and greatest elements to greatest elements.

Another interesting result about $F D(|X|)$ for infinite $X$ is the following.

TheOREM 2. Let $X$ be an infinite set. Then $|F D(|X|)|=\left|2^{2^{x}}\right|$.

Proof (SKeTCH). Clearly, $|F D(|X|)| \leqslant\left|2^{2^{x}}\right|$. Equality will follow once we construct an injection $F: 2^{2^{X}} \rightarrow F D(|X|)$. Since $X$ is infinite there exists a bijection $f:\{1,2\} \times X \rightarrow X$. For $\Gamma \subset X$, let $\Gamma^{*}=\{f(2, x) \mid x \in \Gamma\} \cup$ $\{f(1, x) \mid x \in X-\Gamma\}$. Then an injective $F$ may be defined by $F(S)=\{\Delta \subset$ $X \mid \Delta \subset \Gamma^{*}$ for some $\left.\Gamma \in S\right\}$.

This straightforward answer contrasts strikingly with the difficulty of determining the cardinality of $F D(n)$ for finite $n \geqslant 0$ (see [4]-[6]).

The results in this note appeared in the author's dissertation [6].

\section{REFERENCES}

1. G. Birkhoff, Lattice theory, 3rd ed., Amer. Math. Soc. Colloq. Publ., vol. 25, Providence, R. I., 1967.

2. H. Gaifman, Infinite Boolean polynomials. I, Fund. Math. 54 (1964), 229-250.

3. A. Hales, On the non-existence of free complete Boolean algebras, Fund. Math. 54 (1964), 45-66.

4. D. Kleitman and G. Markowsky, On Dedekind's problem: the number of isotone Boolean functions. II, Trans. Amer. Math. Soc. 213 (1975), 373-390.

5. A. D. Korshunov, A solution to Dedekind's problem on the number of monotone Boolean functions, Dokl. Akad. Nauk SSSR 233 (1977), 543-546. (Russian)

6. G. Markowsky, Combinatorial aspects of lattice theory with applications to the enumeration of free distributive lattices, Ph.D. Thesis, Harvard University, 1973.

7. A. Nerode, Composita, equations and freely generated algebras. Trans. Amer. Math. Soc. 91 (1959), 139-151.

Computer Sciences Department, IBM Thomas J. Watson Research Center, Yorktown HeIGHTS, NeW YORK 10598 